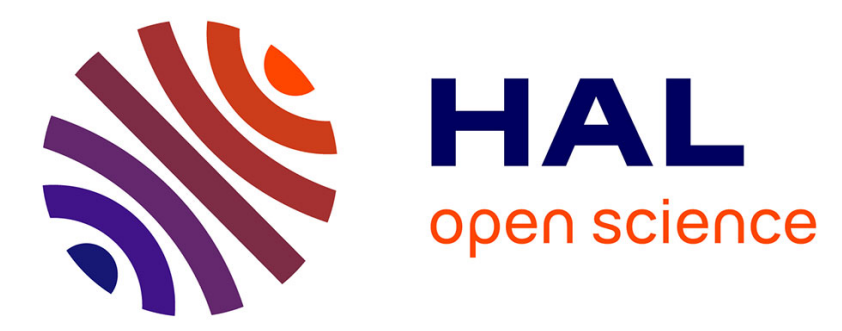

\title{
Analyse et limites de l'évaluation rétrospective d'une concentration
}

Patrice Bougette

\section{To cite this version:}

Patrice Bougette. Analyse et limites de l'évaluation rétrospective d'une concentration. Revue Internationale de Droit Economique, 2009, 23 (2), pp.159-173. hal-00463327

\section{HAL Id: hal-00463327 \\ https://hal.science/hal-00463327}

Submitted on 13 Jan 2011

HAL is a multi-disciplinary open access archive for the deposit and dissemination of scientific research documents, whether they are published or not. The documents may come from teaching and research institutions in France or abroad, or from public or private research centers.
L'archive ouverte pluridisciplinaire HAL, est destinée au dépôt et à la diffusion de documents scientifiques de niveau recherche, publiés ou non, émanant des établissements d'enseignement et de recherche français ou étrangers, des laboratoires publics ou privés. 


\title{
Analyse et limites de l'évaluation rétrospective d'une concentration
}

\author{
Patrice Bougette* \\ Revue internationale de droit économique, vol. 23, $n^{\circ}$ 2, 2009, pp. 159-173
}

\section{Résumé}

Selon une perspective méthodologique, cet article présente les différents moyens d'évaluer une décision de concentration rendue par l'autorité de concurrence. La tendance croissante à mesurer l'efficacité de la politique de concurrence nécessite un point de synthèse des principaux outils existants. Quatre techniques envisageables sont exposées à cette fin : la simulation à l'aide de modèles structurels, l'analyse événementielle des cours boursiers, la méthode des doubles différences et, enfin, les enquêtes et tests de marché. Nous nous demandons dans quelle mesure ces outils habituellement utilisés lors d'une notification de concentration peuvent être transposés pour une étude rétrospective. Les intérêts et limites de chaque approche sont successivement relevés pour souligner leur possible complémentarité.

Mots-clés : Politique de concurrence - Concentrations - Études ex post.

\begin{abstract}
From a methodological perspective, this paper aims to review the various means to assess a competition agency's merger decision. The upward trend to measure the efficiency of competition policy shows the utility of a survey about the main existing quantitative tools. To this end, four different approaches are exposed: simulation with structural models, event studies, the difference-in-difference technique, and lastly, surveys. One can ask if these tools often used during an investigation phase for a merger proposal can be transposed for a retrospective study. The pros and cons of such models are emphasized so that complementarities among them are possible.
\end{abstract}

Keywords: Competition policy - Mergers - Retrospective studies.

JEL Classification: C40, K21, L40.

* Docteur en sciences économiques, LAMETA et Université Montpellier 1. L'auteur tient à remercier Thierry Blayac, Christian Montet et Florent Venayre pour la qualité de leurs commentaires sur une version antérieure de ce texte. E-mail: pbougette@lameta.univ-montp1.fr. 


\section{Introduction}

Simuler l'impact économique d'une concentration est un exercice qui peut normalement s'effectuer aussi bien en amont qu'en aval d'une décision. L'étude en amont est bien sûr la plus courante car elle devient l'outil d'évaluation des effets unilatéraux (hausses de prix potentielles) lors de la notification du projet. L'autorité de la concurrence peut ainsi apprécier au mieux les conséquences quantitatives qu'une acceptation pourrait engendrer, principalement en matière de prix et de parts de marché.

À l'opposé, l'évaluation rétrospective d'une affaire de concentration est plus rare. En effet, elle apparaît moins utile car elle ne peut aboutir à de possibles corrections sur le dossier en question ${ }^{1}$. Pourtant, l'intérêt de telles études, parfois dites post mortem, se situe dans l'élaboration de lignes directrices et, également, dans la capacité à tester la performance des simulations effectuées en amont. Comme le regrette Whinston (2006, p. 127), «l'utilisation de données postérieures à la fusion dans un but d'évaluation des techniques en matière d'analyse prospective des concentrations est malheureusement limitée. 》Si le cas étudié comprend des cessions d'actifs comme engagements, l'intérêt de l'analyse est alors double. La définition du périmètre à céder et le transfert d'actifs méritent en effet un suivi spécifique tant ils ne sont pas exempts de difficultés pratiques (Fréneaux, 2007). Ces synthèses post mortem offrent par ce biais une opportunité de suivi des cessions.

Parmi les simulations d'effets unilatéraux les plus citées, certaines comportent - à des degrés divers - une composante rétrospective. Ainsi, Nevo (2000) utilise des données réelles post-fusion afin de les comparer aux prévisions de son modèle de simulation. Dans l'industrie des céréales du petit déjeuner, ses prédictions se révèlent proches des variations effectives de prix, à l'exception d'un cas dont l'auteur attribue l'écart à la forte concurrence hors prix qui n'a pas été prise en compte dans la modélisation. Pinkse et Slade (2004) simulent également deux cas de concentration dans l'industrie de la bière en Angleterre ${ }^{2}$. La fusion Courage/Scottish, montrent-ils, a eu très peu d'effet sur les prix, moins de $1 \%$ de hausse. En revanche, la fusion Bass/Carlsberg-Tetley aurait provoqué une augmentation des prix de $3 \%$ si l'autorité ne l'avait pas rejetée. L'étude de Peters (2006) propose également une analyse rétrospective de cinq fusions sur le marché aérien transatlantique entre 1986 et 1987 . Contrairement aux travaux de Pinske et Slade (2004), l'auteur montre que les différences obtenues entre les prix effectifs post-fusion et les variations simulées sont importantes. Il ne faut pas forcément y voir une incapacité intrinsèque de leur méthode à prédire correctement les variations de prix mais plutôt dans ce cas, semble-t-il, le choix d'une concurrence en prix peu approprié à la dynamique du marché aérien ${ }^{3}$.

Récemment, Ashenfelter et Hosken (2008) réalisent une étude a posteriori de cinq concentrations acceptées dans l'industrie du commerce de détail aux États-

\footnotetext{
1. Sur la question de l'efficacité du contrôle des concentrations, voir Bougette et Venayre (2008) qui analysent les liens entre contrôle ex ante et contrôle ex post.

2. Les fusions étudiées sont celle de Courage/Scottish and Newcastle autorisée en 1995 et celle de Bass/Carlsberg and Tetley finalement interdite en 1997. Les données utilisées datent de 1995 et rendent ainsi possibles deux types de scenario : i) revenir sur la fusion Courage/Scottish, ii) prédire les effets de la fusion Bass/Carlsberg and Tetley.
}

3. Nous reviendrons plus loin sur l'importance de la forme de concurrence choisie. 
Unis ${ }^{4}$. Ce secteur se prête bien à ce type d'étude en raison de la disponibilité de données scannées qui sont collectées et traitées par des sociétés spécialisées. Dans ce cas précis, les affaires choisies sont celles les plus susceptibles d'avoir entraîné des hausses de prix en raison de l'importance des problèmes concurrentiels soulevés lors de leur notification. Les résultats des auteurs sont clairs : quatre des cinq fusions étudiées ont entraîné des hausses de prix significatives comprises entre $3 \%$ et $7 \%$; seule l'affaire Aurora/Log Cabin ne revêt aucune tendance haussière sur les années qui ont suivi la décision d'acceptation.

L'objectif de ce travail est de dresser un bilan des principales méthodes quantitatives afin de mesurer l'effet d'une concentration horizontale. L'accent est mis sur l'évaluation rétrospective. Une simple transposition des modèles qui servent en amont est-elle possible? Quels sont les risques inhérents à ce type d'analyse? Nous verrons qu'il existe quatre principales techniques adaptables : la simulation des effets à partir d'un modèle structurel, l'analyse événementielle des cours boursiers des entreprises, la méthode des doubles différences et, pour finir, l'élaboration d'enquêtes envoyées aux parties à la concentration ainsi qu'aux autres acteurs du marché. Nous nous proposons de les détailler successivement tout en montrant leur efficacité respective lorsqu'il s'agit d'évaluer ex post une concentration.

\section{La simulation des effets unilatéraux}

La méthode de simulation des effets d'une fusion s'inscrit traditionnellement dans la modélisation des interactions entre oligopoleurs. La simulation peut être effectuée ex post afin de comparer les effets prix constatés sur le marché avec la prévision effectuée au moment même de la décision. Une attention particulière doit être portée à la qualité des données postérieures à la fusion. En effet, ces données sont le fruit de la fusion elle-même mais également du résultat de la décision.

\subsection{Quel type de concurrence sur le marché?}

Il faut bien reconnaître qu'en général la simulation de fusion s'emploie plus volontiers dans des cas de concurrence en prix (type Bertrand) que dans des cas de concurrence en capacités, d'autres alternatives étant toutefois possibles (Werden et Froeb, 2008). Au moins deux raisons peuvent être avancées pour expliquer la prédominance de la concurrence en prix. D'une part, ce type de concurrence est en définitive beaucoup plus fréquent que la concurrence en quantités. Il concerne principalement une concurrence inter-marque ( $c f$. infra). D'autre part, en termes techniques, il est possible de donner une valeur aux coûts marginaux de production directement à partir des conditions de premier ordre du programme de maximisation du profit des entreprises. Les recherches ultérieures se sont davantage portées sur le raffinement du type de demande plutôt que sur les diverses formes de concurrence sur le marché. Deux alternatives sont néanmoins possibles.

4. Les fusions sont les rachats de Quaker State par Pennzoil, de Tambrands par Procter and Gamble, des céréales Chex par General Mills, de Log Cabin par Aurora, enfin de la concentration entre Guinness et Grand Metropolitan. 
Une concurrence en quantités (type Cournot) est principalement caractérisée par des investissements en capacité de production élevés ${ }^{5}$. Le marché automobile est un exemple classique de ce type de concurrence, mais d'autres secteurs tels que l'énergie, l'acier ou bien encore l'aérien rentrent dans cette catégorie. En matière de simulations, Lundmark et Nilsson (2003) calculent les effets d'une fusion sous ce type de concurrence. Ils prennent l'exemple du cas européen $C V R D / C a e m i$, fusion de deux entreprises productrices de minerai de fer, acceptée en 2003 par la Commission ${ }^{6}$. Les auteurs confirment les conséquences négatives du rapprochement sur le bien-être social et justifient ainsi les engagements acceptés dès la Phase 1. En réalité, un nombre non négligeable d'entreprises fonctionnent en choisissant d'abord leurs capacités, puis ensuite se lancent dans une concurrence en prix. Dans ce cas, Friedman (1988) montre l'équivalence avec une concurrence à la Cournot.

Néanmoins, comme le résument Kaplow et Shapiro dans le Handbook of Law and Economics de 2007, «le modèle de Cournot n'est pas approprié lorsque les industries comportent des produits fortement différenciés ${ }^{7}$. » En effet, lorsque la différenciation des produits est très forte, le modèle de Cournot obtient des résultats similaires à celui d'un modèle Bertrand différencié. Les prix en Cournot sont en général plus élevés que les prix en Bertrand ceteris paribus, mais lorsque les produits sont suffisamment différenciés, l'écart entre les prix s'estompe (Singh et Vives, 1984; Häckner, 2000). La forte différenciation des produits l'emporte sur la concurrence en quantités.

Enfin, un dernier cadre théorique est également envisageable. Il s'agit d'un modèle d'enchères, par exemple pour des marchés publics avec appels d'offres. Cette littérature est plus récente et s'adapte à de nombreuses affaires. En matière de simulations, les travaux de Tschantz et al. (2000) et Dalkir et al. (2000) permettent de quantifier les effets d'une fusion à l'aide d'un modèle d'enchères au premier prix $^{8}$. Le modèle d'enchères descendantes de Waehrer et Perry (2003) est plus performant dans la mesure où il autorise l'asymétrie des participants : les entreprises supportent des coûts différents et ont donc des capacités différentes. Les auteurs montrent qu'une fusion affaiblit généralement la position de l'acheteur, bien qu'il soit à même de modifier son prix de réserve afin d'en atténuer l'effet. Les modèles d'enchères ont par exemple été utilisés avec succès par la Commission lors de la notification de la fusion Oracle/PeopleSoft ${ }^{9}$.

5. Une des dernières décisions de la DGCCRF qui mentionne explicitement une concurrence Cournot est celle concernant l'affaire Pan Fish/Marine Harvest dans le secteur de la production et de la vente de saumon (lettre C2006-47 du $1^{\mathrm{er}}$ déc. 2006).

6. Déc. Comm. CE n COMP M.3161, 18 juil. 2003.

7. Kaplow et Shapiro (2007, p. 1143).

8. On distingue habituellement quatre types d'enchères. Dans les enchères ascendantes, le prix augmente jusqu'à ce qu'il ne demeure plus qu'un seul enchérisseur, qui remporte l'enchère au dernier prix. Dans les enchères descendantes, le prix diminue jusqu'à ce qu'un candidat se déclare preneur et remporte les enchères au dernier prix. Dans les enchères sous pli scellé au premier prix, chaque enchérisseur remet une offre sans connaître les autres soumissions. Le bien est attribué au plus offrant, qui paie le montant offert. Dans des enchères sous pli scellé au deuxième prix, chaque enchérisseur remet une offre sans connaître les autres soumissions. Le bien est attribué au plus offrant, qui paie le prix offert par le deuxième plus offrant (OCDE, 2006).

9. Déc. Comm. CE no COMP./M.3216, 26 oct. 2004. Cette concentration dans le secteur des logiciels avait également donné lieu à une enquête des autorités américaines qui, initialement opposées, avaient dû donner leur accord après le jugement devant la District Court of Northern California. Des divergences portaient sur le choix du modèle d'enchères et la vali- 


\subsection{Le choix d'un système de demande}

Il faut ensuite choisir le type demande qui correspond le mieux aux produits en question. Cette étape est délicate car le type de demande affecte de manière significative les prévisions post-fusion (Crooke et al., 1999). La forme de demande la plus répandue est une distribution logit, ce qui a donné le nom au Modèle Logit Antitrust (Werden et Froeb, 1994). Le succès de cette forme de demande tient à sa simplicité d'estimation. En revanche, elle impose des restrictions sur le type de substituabilité entre les marques. Il s'agit de la propriété IIA $^{10}$. Deux nouvelles versions du modèle sont apparues dans la littérature pour introduire plus de flexibilité. Une première extension a intégré des groupes de produits; les produits à l'intérieur d'un groupe sont plus substituables entre eux qu'envers les autres produits. Ce nouveau modèle, appelé logit emboîté, a été par exemple appliqué à la concentration de Volvo/Scania (Ivaldi et Verboven, $2005)^{11}$ et à celle de Lagardère/Natexis/VUP ${ }^{12}$ (Ivaldi, 2005). Le logit mixte, seconde variante, possède une demande qui intègre un plus grand nombre de caractéristiques (Nevo, 2000). Cette flexibilité accrue ne s'obtient pas sans coût car les exigences en termes de données et de calculs sont beaucoup plus fortes.

La seconde classe de demande utilisée est celle nommée AIDS (pour Almost Ideal Demand System). L'étude réalisée par Hausman et al. (1994) constitue certainement l'application la plus célèbre de cette forme fonctionnelle. Il est clair que le choix de la forme de la demande dépend bien souvent du type de données recueillies lors des différents tests de marché et des caractéristiques du produit. Une version simplifiée du modèle, le PCAIDS, repose sur le principe de la proportionnalité qui permet de réduire le nombre de paramètres nécessaires à la simulation (Epstein et Rubinfeld, 2002). L'hypothèse de proportionnalité signifie que la perte de parts de marché d'une marque suite à l'augmentation de son prix est reportée sur les marques concurrentes en proportion de leurs parts de marché respectives. Le nombre florissant d'études empiriques qui utilisent le modèle PCAIDS est une preuve de sa grande popularité (Davies et Lyons, 2007). Signalons, par ailleurs, que plusieurs autorités ont officiellement adopté l'outil. Il apparaît en effet explicitement dans les textes de leurs décisions (l'Office of Fair Trading anglais et l'autorité de concurrence italienne notamment).

Pour ce qui concerne la mesure de la substituabilité entre produits, il est pratique de calculer les ratios de diversion ${ }^{13}$, c'est-à-dire la part de la demande de la marque dont le prix a augmenté et qui est reportée vers des produits concurrents (Shapiro, 1996). Les autorités doivent veiller à ce que les produits commercialisés par les parties notifiantes ne conduisent pas à des ratios de di-

dité de certaines données. Preston McAfee avait été, pour cette occasion, expert auprès du Department of Justice américain.

10. La propriété IIA (Independance from Irrelevant Alternatives) implique que le rapport des probabilités de choix entre deux produits ne dépend que des caractéristiques de ces deux produits. Ajouter ou enlever des produits dans l'ensemble de choix ne doit pas modifier le rapport des probabilités de choix des deux produits en question.

11. Déc. Comm. CE n ${ }^{\circ}$ COMP./M.1672, 15 mars 2000. Sur cette affaire, les parties fusionnant ont commandé une contre-expertise (Hausman et Leonard, 2005).

12. Déc. Comm. CE n ${ }^{\circ}$ COMP./M.2978, 7 janv. 2004.

13. Il s'agit de la traduction courante de l'expression anglaise diversion ratio. Une expression plus conforme à l'idée de départ serait certainement « ratio de détournement ». En France, ces ratios ont pour la première fois été cités lors des affaires Tabur $/ M r$ Bricolage (2002) et COMAREG/France Antilles (2003). 
version trop faibles, de telle sorte qu'un report de la demande vers les marques concurrentes puisse réellement exister.

\subsection{Calibrage ou estimation du modèle}

Après avoir choisi la forme de la demande et le type de concurrence, deux voies sont alors possibles pour parvenir à l'équilibre offre-demande. La première est l'estimation qui consiste à déterminer les valeurs des paramètres d'un modèle dans le but d'obtenir la meilleure approximation du processus qui a généré les données économiques. Il s'agit du cas idéal lorsque le chercheur a en sa possession une base de données exhaustive et peut ainsi construire un modèle économétrique de l'industrie (Nevo, 2000; Pinkse et Slade, 2004; Dubé, 2005). Quand les données ne sont pas disponibles ou sont incomplètes, la solution alternative est de recourir au calibrage. Cette méthode conduit à assigner aux paramètres du modèle des valeurs qui sont les plus pertinentes compte tenu de l'information disponible (Werden et Froeb, 1994). Le calibrage permet une bonne approximation des effets en jeu mais ne fournit aucune mesure de robustesse ou de significativité des résultats contrairement à l'estimation d'un modèle (tests statistiques sur les paramètres) ${ }^{14}$.

La technique du calibrage revient à choisir les valeurs des paramètres pour que le modèle puisse refléter certains traits de l'industrie qui auraient prévalu dans un futur proche en l'absence de fusion. Une bonne approximation consiste à utiliser les prix et les parts de marché sur une période récente avant la fusion (en général un an, suivant les données disponibles). Les élasticités-prix de la demande sont souvent estimées à l'aide d'un modèle économétrique en utilisant des données microéconomiques ou des résultats d'enquêtes. Parfois, dans le cas de données issues de « scanning », l'estimation peut poser des problèmes et aboutir à des résultats peu convaincants ${ }^{15}$. Dans ce dernier cas, les élasticités-prix de la demande doivent être encadrées sur des intervalles à l'aide d'indications de préférence, d'études marketing ou tout autre type de documentation.

Les effets prix prévus sont les différences entre les prix post-fusion simulés et les prix observés. Simuler les prix post-fusion requiert les valeurs des coûts marginaux pour tous les produits considérés dans la simulation. Ces valeurs sont généralement calculées à partir du modèle calibré : les coûts marginaux pré-fusion sont choisis de telle manière que les prix et les parts de marché constituent un équilibre en prenant en compte les élasticités-prix. En calculant le prix post-fusion, on suppose que les coûts marginaux sont invariants à la quantité produite. Enfin, le bien-être est calculé pré- et post fusion. Il constitue la somme des profits de l'industrie et du surplus des consommateurs. Selon la variation de bien-être induite par la concentration, les autorités de concurrence évaluent le caractère pro- ou anticoncurrentiel de l'opération (bien souvent, la seule variation des prix leur suffit).

14. La première méthode estime une forme réduite d'un modèle avec peu de paramètres, souvent basé sur la théorie; la seconde calibre un modèle comprenant un ensemble important de paramètres et simule à partir de données pertinentes.

15. Le scanning est un procédé utilisé dans la grande distribution pour récolter les prix et ventes des produits grâce aux codes barres. Cf. l'analyse de Hosken et al. (2002) pour les problèmes induits par ce type de données. 


\section{L'analyse événementielle des cours boursiers}

Le cours boursier des entreprises fusionnant et de ses rivales fournit des indications sur le caractère concurrentiel de l'opération ${ }^{16}$. Ainsi, dans le cas où la concentration correspond à une stratégie anticoncurrentielle, on peut supposer que les opérateurs boursiers vont anticiper les profits que l'accroissement du pouvoir de marché de la nouvelle entité permettra de réaliser. Par conséquent, le jour de l'annonce ou peu de temps après, le cours boursier des entreprises fusionnant devrait s'élever. Cependant, on doit aussi s'attendre à ce que les titres de la plupart des autres firmes du secteur les accompagnent. En effet, si la concentration projetée a effectivement pour effet de réduire l'intensité de la concurrence, toutes les firmes du même secteur en profiteront. À l'inverse, lorsque la fusion est à la source de synergies bénéfiques pour les consommateurs, les cours des actions des entreprises souhaitant fusionner prendront de la valeur dès l'annonce de la fusion, mais n'entraîneront pas les titres des autres entreprises dans leur sillage. On a alors toute raison de penser que la concentration est davantage liée à la naissance d'un opérateur plus efficace, plutôt qu'à l'existence de pratiques restrictives.

Un calendrier précis des dates clefs de l'opération doit être établi : dates de l'annonce du projet, de la notification, de la décision de l'autorité, et parfois date des engagements requis. Cette modélisation économétrique a un avantage non négligeable : les données qu'elle requiert sont généralement disponibles sans coût et permettent de comparer rapidement les situations alternatives à la situation effective du marché.

Cependant, quelques difficultés liées soit à la mise en œuvre de la méthode, soit à l'interprétation des résultats, méritent d'être détaillées. En premier lieu, les entreprises concernées par l'opération doivent être cotées en bourse. La méthode s'applique donc uniquement à des opérations d'envergure ${ }^{17}$. On peut toutefois la mettre en œuvre même si toutes les entreprises ne sont pas cotées sur le marché, les résultats concernant les firmes cotées n'en sont pas altérés. C'est ce qu'a choisi de faire le rapport du LEAR (2006), à partir du moment où les principaux concurrents de Pirelli et BICC étaient cotés en bourse. Les auteurs concluent :

«En moyenne, les concurrents n'ont pas bénéficié de la fusion, contrairement aux clients. Par conséquent, nous pouvons dire que le marché financier s'attendait à ce que la fusion soit pro-concurrentielle et la Commission a eu raison de l'autoriser ${ }^{18}$. »

Cette méthode suppose par ailleurs que l'annonce de l'opération n'ait été précédée d'aucune rumeur ou anticipation afin de s'assurer que la variation du cours boursier traduise uniquement le caractère concurrentiel de l'opération (Janin et Menoni, 2007). En cas d'anticipation, en effet, certaines fusions anticoncurrentielles pourraient réduire le cours boursier des concurrents, tout en accroissant leur profit, posant ainsi des problèmes d'interprétation (Fridolfsson et Stennek, 2006). Or, la plupart des fusions d'envergure se passent rarement

16. Pour une revue de la littérature, voir Duso et al. (2007).

17. Pour une application à la fusion Carrefour/Promodès, cf. Janin et Menoni (2007).

18. Lear (2006, p. 101). Pour une analyse détaillée de la méthodologie proposée dans le rapport, $c f$. Bougette (2007). 
sans aucune rumeur sortie dans la presse. Le cours boursier apparaît ainsi limité pour ne pas déformer l'information concurrentielle issue du rapprochement.

Enfin, si le cours boursier des concurrents ne réagit pas, il ne faut pas forcément en conclure d'une manière hâtive que la fusion n'entraîne aucune hausse de prix. Parfois, comme le démontrent McAfee et Williams (1988), lorsque les entreprises rivales sont des conglomérats et par conséquent reçoivent une faible part de profit issu du marché en question, le cours boursier n'est pas forcément altéré; le test en devient donc moins performant. Mullin et al. (1995) proposent alors une alternative : s'intéresser aux cours boursiers des clients des parties fusionnant en lieu et place des concurrents. La réaction des clients par l'intermédiaire de leurs cours boursiers permet également d'avoir une indication sur l'aspect anticoncurrentiel ou non de la fusion. Cette analyse, cependant, ne fonctionne pas dans le cas où les clients sont eux-mêmes producteurs car ils pourraient reporter la hausse des prix de leurs fournisseurs sur les consommateurs finals.

Finalement, Neven et Zenger (2008) de la Commission européenne mettent en garde sur les dérives possibles de ces études : « si les autorités de la concurrence prennent comme base d'évaluation sérieuse les études événementielles, elles sont alors incitées à suivre la grille de lecture que le marché propose de la concentration plutôt que de mener leurs propres investigations ${ }^{19}$. » Cette approche constitue davantage une analyse complémentaire mais en aucun cas ne doit se suffire à elle-même pour juger du bien-fondé d'une concentration.

\section{La méthode des doubles différences}

Les méthodes d'évaluation des programmes sont un patchwork de différentes techniques d'estimation qui permettent de comparer le comportement de deux groupes d'agents : le groupe de contrôle (ou de référence) et le groupe expérimental (testé). L'idée sous-jacente est de mesurer, ceteris paribus, la différence de performance entre les deux groupes, qui sera de fait une estimation de l'effet de la décision, dans notre cas celle de l'autorité de concurrence.

Plus spécifiquement, la méthode d'évaluation la plus couramment employée en matière d'évaluation rétrospective est appelée les « doubles différences ${ }^{20}$. Elle consiste à analyser des chocs d'offre et de demande en utilisant les prix sur des marchés géographiques non affectés. Ainsi, la comparaison permet d'avoir une approximation de ce que les prix auraient été sur les marchés affectés si la fusion n'avait pas eu lieu. Les effets sont alors mesurés comme la variation des prix suite à la fusion dans une région affectée par rapport à la variation de prix dans la région de contrôle.

L'étude menée par Hastings (2004) constitue, sans aucun doute, l'application de référence de cette méthode dans le domaine antitrust. Elle s'intéresse à la mise en place de contrats de licence à long terme entre les raffineries Arco et 260 stations services dans le sud de la Californie. En examinant les prix de vente des stations services sur des régions voisines, Hastings parvient à montrer que la suppression du seul distributeur indépendant des différents marchés affectés

19. Neven et Zenger (2008, p. 487).

20. Pour un exposé de la technique, $c f$. Imbens et Wooldridge (2009). 
entraînerait une hausse des prix. Ce même procédé a été repris dans des cas de concentrations. Taylor et Hosken (2007), tous deux économistes à la FTC, estiment les effets prix (de vente et de gros) de la création de l'entreprise commune Marathon/Ashland Petroleum également dans l'industrie pétrolière. Leurs résultats indiquent que le rapprochement n'a pas poussé les prix de vente à la hausse, contrairement aux plaintes et critiques de l'opinion publique à l'époque de l'affaire ${ }^{21}$. Une évaluation récente, dont les conclusions sont plus sévères à l'égard de l'autorité, est celle de Tenn (2008). L'attention de l'économiste de la FTC s'est portée sur l'acquisition en 1999 de Summit, un réseau d'hôpitaux dans la banlieue de San Francisco, par Sutter un réseau concurrent dans le même secteur. L'utilisation de l'approche par les doubles différences permet à l'auteur d'établir que la transaction a provoqué une hausse substantielle du prix des services proposés par Summit.

Pour le cas français, Choné et Linnemer (2008) s'appuient sur une approche analogue. Ils analysent les effets unilatéraux de la concentration Vinci/GTM ${ }^{22}$ en France. Cette fusion des deux grands groupes a eu un impact sur plusieurs marchés, notamment celui sur lequel les auteurs se sont penchés, les parkings dans la région parisienne. La base de données utilisée ne concerne que les parkings publics payants ${ }^{23}$. Leurs résultats attestent d'une hausse moyenne des prix « modérée ».

Cette méthode s'avère performante et permet de tester la robustesse des résultats. En revanche, il n'est pas toujours aisé d'identifier un bon groupe de contrôle. En effet, il est nécessaire de trouver des produits qui appartiennent à des marchés géographiques différents, donc non affectés par la concentration, et qui font en même temps face aux mêmes conditions de coûts et de demande. Des conditions restrictives qui parfois ne permettent pas de trouver un groupe de contrôle approprié.

\section{Les enquêtes via l'envoi de questionnaires}

Un dernier outil utilisé par les autorités consiste à mener une enquête de marché à partir de questionnaires. Il s'agit vraisemblablement du moyen le plus flexible pour obtenir des données et une vision d'ensemble d'une concentration d'entreprises. Une enquête nécessite la collecte de données à partir d'un échantillon représentatif des participants au marché pertinent, à travers un questionnaire écrit ou des entretiens avec des personnes directement impliquées dans l'opération. Le questionnaire peut être potentiellement envoyé aux concurrents et aux clients, en fonction du nombre d'acteurs en jeu. Le procédé est souvent utilisé en amont de la décision, lors du test de marché, pour sonder les réactions des tiers au rapprochement des entreprises notifiantes.

21. Simpson et Taylor (2008) étudient une affaire sur ce même type de marché. Ils parviennent à la même conclusion : aucune preuve de hausse des prix finals.

22. Avis $\mathrm{n}^{\circ} 01-\mathrm{A}-08$ du 5 juin 2001 relatif à l'acquisition du Groupe GTM par la société Vinci. Concentration acceptée avec une injonction au terme d'une Phase 2.

23. Ces parkings publics représentent environ la moitié de l'offre totale de parkings dans la capitale. 


\subsection{Méthodologie spécifique à une évaluation ex post}

Pour rassembler les données nécessaires à une étude ex post, les chercheurs ont la possibilité d'élaborer un questionnaire spécifique à l'opération et de l'envoyer aux différents acteurs du marché. Le rapport du LEAR (2006) propose l'envoi de deux questionnaires aux parties concernées par l'opération. Le premier questionnaire traite de questions générales à l'opération : délimitation du marché pertinent, du type de concurrence, des effets unilatéraux et coordonnés, les synergies attendues et quelques questions ouvertes. Le second questionnaire essaie d'identifier des arguments-clés qui manquent à l'analyse, le plus souvent par l'intermédiaire de questions de type réponse courte.

Ensuite, l'analyse des résultats est cruciale. Il est conseillé de procéder à une analyse croisée des différentes questions, voire à une analyse de données (analyse en composantes principales, analyse factorielle, analyse discriminante, réseaux de neurones) pour faire apparaître des interactions plus fines.

En pratique, un compromis doit être trouvé entre le maximum d'informations à collecter et la simplicité du questionnaire pour les entreprises. Rappelons que, selon les textes actuels, ces dernières ne sont pas tenues d'y répondre.

\subsection{Des biais méthodologiques inhérents à l'outil}

Les questionnaires sont néanmoins soumis à deux biais méthodologiques dont il convient de ne pas négliger l'importance, tant pour ce qui concerne l'évaluation ex ante qu'ex post d'une concentration. Premièrement, l'autorité s'expose à un faible taux de retour des questionnaires. Aussi bien pour des raisons de logistique, de contrainte de temps, ou encore de crainte de divulgation des données confidentielles, les entreprises peuvent rechigner à remplir et retourner le questionnaire. À titre illustratif, Reynolds et Walters (2008) font un bilan des questionnaires clients utilisés par l'autorité de concurrence anglaise, la Competition Commission, et obtiennent des taux de retour des questionnaires ne dépassant pas en général $50 \%$. Dans le cas d'une étude rétrospective, il est donc nécessaire que l'autorité envoie elle-même le questionnaire même si un organisme extérieur est chargé de l'étude. Elle doit également privilégier sur certains aspects des entretiens qui apparaissent moins pénibles qu'un questionnaire. Comme ils sont susceptibles de représenter un surcoût pour l'autorité, ils doivent rester peu nombreux et ciblés sur quelques personnes-clés de l'opération.

La deuxième limite méthodologique est naturellement liée à la subjectivité des réponses déclarées lors du test de marché. Elles ne reflètent pas forcément l'opinion réelle des protagonistes. Les questionnés sont incités soit à répondre favorablement à l'autorité, soit à dissimuler des faits qui ne correspondraient pas à son attente. En particulier, dans le cas de clients, même s'ils constituent «les acteurs du marché les moins biaisés car ils sont en position de perdre le plus d'une fusion anticoncurrentielle », plusieurs limites apparaissent dans la prise en compte de leurs avis (Tucker et al., 2007). D'abord, les clients peuvent manquer de connaissances de bases sur les marchés impliqués par la concentration. Il leur est par conséquent difficile de porter un jugement. Le choix d'un groupe de clients suffisamment représentatifs est ensuite problématique. Il peut s'agir de groupes minoritaires qui tentent de capter l'autorité pour des intérêts 
spécifiques. Il faut avant tout s'assurer de l'indépendance des clients vis-à-vis des parties fusionnant lorsqu'il s'agit de firmes.

Du côté des fournisseurs, leurs réponses au test de marché doivent également faire l'objet de précaution. Le premier effet probable tend à une surréaction de leur part : la puissance d'achat accrue de l'entité fusionnée peut inciter les fournisseurs à facilement condamner le rapprochement en question de peur de voir leur pouvoir de négociation affaibli. Le second effet vise au contraire à sous-évaluer les effets anticoncurrentiels probables. Les fournisseurs risquent de ne pas donner leur réel avis sur la concentration de peur que l'entreprise une fois fusionnée ne se détourne d'eux. L'effet net entre ces réactions opposées n'est pas tranché et doit être appréhendé à la lumière des éléments du dossier.

$\mathrm{Au}$ final, le poids à accorder aux tiers fait encore débat. Une scrupuleuse analyse de données des résultats de l'enquête peut aider à déceler de tels comportements aberrants; il existe néanmoins une part de doute sur la qualité des réponses collectées.

\section{Conclusion}

La quantité de données nécessaires afin de simuler une concentration est parfois un frein à l'évaluation ex post. Toutefois, contrairement au cas ex ante où le délai est court pour recueillir des données complètes et les plus désagrégés possibles, dans le cas d'une évaluation rétrospective cette contrainte est assouplie. Il n'en demeure pas moins que différentes catégories de données sont indispensables : les prix, les quantités ou parts de marché, les données sur les coûts tels que le prix des inputs, des informations sur les caractéristiques liées aux produits. Toutefois, ces données peuvent être onéreuses si elles sont acquises par l'intermédiaire de sociétés spécialisées dans la collecte de données. Ainsi, une simulation peut être couplée avec des enquêtes auprès des principaux acteurs afin d'obtenir des données à moindre coût.

Il est souhaitable que davantage de cas individuels de concentration soient traités par les différentes autorités. En revanche, l'idée d'allouer les propres ressources de l'autorité en fonction de l'efficacité de ses décisions ne semble pas souhaitable. Neven et Zenger (2008) détaillent en effet plusieurs dérives qui résulteraient d'une telle allocation. D'une part, ce type d'évaluation ex post favoriserait plus les autorités qui seraient plutôt « interventionnistes » que « laxistes » dans la mesure où il y aurait moins de chances de constater des hausses des prix suite à des décisions plus sévères. D'autre part, l'autorité pourrait être amenée à négliger tout type d'information qui serait susceptible de mettre en lumière une « mauvaise » décision.

L'esprit de l'évaluation ex post est de se nourrir des décisions rendues afin de satisfaire au mieux les notifications des projets de concentration tout en se préservant des méfaits potentiels de certains regroupements. L'étude de l'impact des décisions sur le marché est alors d'une incroyable richesse dont il serait dommage que les autorités se privent. 


\section{Références}

[1] Ashenfelter O. et D. Hosken (2008), "The Effect of Mergers on Consumer Prices : Evidence from Five Selected Case Studies", Document de travail, $\mathrm{n}^{\circ} 13859$, NBER, mars 2008. À paraître dans Journal of Law and Economics.

[2] Bougette P. (2007), "Vers un développement d'études ex post en matière de concentration", Revue Lamy de la concurrence : droit, économie, régulation, vol. 12 , p. $165-169$.

[3] Bougette P. et F. Venayre (2008), "Contrôles a priori et a posteriori des concentrations : comment augmenter l'efficacité des politiques de concurrence?", Revue d'économie industrielle, vol. 121, p. 9-40.

[4] Choné P. et L. Linnemer (2008), "Have Parking Prices Risen in Paris? A Retrospective Merger Study", mimeo, $35^{\mathrm{e}}$ conférence de l'EARIE, Toulouse.

[5] Crooke P., L.M. Froeb, S. Tschantz et G.J. Werden (1999), "The Effects of Assumed Demand Form on Simulated Postmerger Equilibria", Review of Industrial Organization, vol. 15, n⿳⺈ 3, p. 205-217.

[6] Dalkir S., J.W. Logan et R.T. Masson (2000), "Mergers in Symmetric and Asymmetric Noncooperative Auction Markets : The Effects on Prices and Efficiency", International Journal of Industrial Organization, vol. 18, $\mathrm{n}^{\circ} 3$, p. $383-413$.

[7] Davies S. et B.R. Lyons (2007), Mergers and Merger Remedies in the EU : Assessing the Consequences for Competition, Londres : Edward Elgar.

[8] Dubé J.-P. (2005), "Product Differentiation and Mergers in the Carbonated Soft Drink Industry", Journal of Economics and Management Strategy, vol. $14, \mathrm{n}^{\circ} 4$, p. 879-904.

[9] Duso T., D.J. Neven et L.-H. Röller (2007), "The Political Economy of European Merger Control : Evidence using Stock Market Data", Journal of Law and Economics, vol. 50, n 3 , p. 455-489.

[10] Epstein R.J. et D.L. Rubinfeld (2001), "Merger Simulation : A Simplified Approach with New Applications", Antitrust Law Journal, vol. 63, n 3 , p. $883-920$.

[11] Fréneaux L. (2007), "L'efficacité du recours aux engagements en matière de contrôle des concentrations", Revue internationale de droit économique, vol. $21, \mathrm{n}^{\mathrm{O}} 1$, p. $43-67$.

[12] Fridolfsson S.-O. et J. Stennek (2006), "Industry Concentration and Welfare - On the Use of Stock Market Evidence from Horizontal Mergers", Document de travail, Discussion Papers n ${ }^{\circ} 5977$, CEPR. À paraître dans Economica.

[13] Friedman J.W. (1988), "On the Strategic Importance of Prices versus Quantities", RAND Journal of Economics, vol. 19, n 4, p. 607-622.

[14] Häckner J. (2000), "A Note on Price and Quantity Competition in Differentiated Oligopolies ", Journal of Economic Theory, vol. 93, p. 233-239.

[15] Hastings J.S. (2004), "Vertical Relationships and Competition in Retail Gasoline Markets : Empirical Evidence from Contract Changes in Southern California", American Economic Review, vol. 94, nº 1, p. 317-328. 
[16] Hausman J.A. et G.K. Leonard (2005), "Using Merger Simulation Models : Testing the underlying assumptions", International Journal of Industrial Organization, vol. 23, n ${ }^{\circ}$ 9-10, p. 693-698.

[17] Hausman J.A., G.K. Leonard et J.D. Zona (1994), "Competitive Analysis with Differentiated Products", Annales d'économie et de statistique, vol. 34, p. $159-180$.

[18] Hosken D., D. O'Brien, D. Scheffman et M. Vita (2002), "Demand System Estimation and its Application To Horizontal Merger Analysis" in The Use of Econometrics in Antitrust. Harkrider J. éd. : American Bar Association Section on Antitrust.

[19] Imbens G.W. et J.M. Wooldridge (2009), "Recent Developments in the Econometrics of Program Evaluation", Journal of Economic Literature, vol. 47, $\mathrm{n}^{\mathrm{o}} 1$, p. $5-86$.

[20] Ivaldi M. (2005), "Mergers and the New Guidelines : Lessons from Hachette-Editis" in Modelling European Mergers. Theory, Competition Policy and Case Studies. van Bergeijk P.A. et Kloosterhuis E. éds. Cheltenham : Edward Elgar, p. 92-106.

[21] Ivaldi M. et F. Verboven (2005), "Quantifying the Effects from Horizontal Mergers in European Competition Policy", International Journal of Industrial Organization, vol. 23, no 9-10, p. 669-691.

[22] Janin L. et B. Menoni (2007), "Le contrôle des concentrations en France : une analyse empirique des avis du Conseil de la concurrence", Économie et prévision, vol. 178-179, $\mathrm{n}^{\mathrm{o}} 2-3$, p. 93-114.

[23] Kaplow L. et C. Shapiro (2007), "Antitrust" in Handbook of Law and Economics, Volume 2. Polinsky A.M. et S. Shavell éds. : Elsevier, p. 1073-1225.

[24] Lear (2006), Ex Post Review of Merger Control Decisions, Rapport pour la Direction Concurrence, Commission européenne.

[25] Lundmark R. et M. Nilsson (2003), "What Do Economics Simulations Tell Us? Recent Mergers in the Iron Ore Industry", Resources Policy, vol. 29, p. $111-118$.

[26] McAfee R.P. et M.A. Williams (1988), "Can Event Studies Detect Anticompetitive Mergers?", Economics Letters, vol. 28, p. 199-203.

[27] Mullin G.L., J.C. Mullin et W.P. Mullin (1995), "The Competitive Effects of Mergers : Stock Market Evidence from the U.S. Steel Dissolution Suit", RAND Journal of Economics, vol. 26, no 2, p. 314-330.

[28] Neven D.J. et H. Zenger (2008), "Ex Post Evaluation of Enforcement : A Principal-Agent Perspective", De Economist, vol. 156, p. 477-490.

[29] Nevo A. (2000), "Mergers with Differentiated Products : The Case of the Ready-to-Eat Cereal Industry", RAND Journal of Economics, vol. 31, $\mathrm{n}^{\mathrm{o}} 3$, p. 395-421.

[30] OCDE (2006), "Competition in Bidding Markets", Document de travail, COMP.(2006)31.

[31] Peters C. (2006), "Evaluating the Performance of Merger Simulation : Evidence from the U.S. Airline Industry", Journal of Law and Economics, vol. $49, \mathrm{n}^{\circ} 2$, p. 627-649. 
[32] Pinkse J. et M.E. Slade (2004), "Mergers, Brand Competition, and the Price of a Pint", European Economic Review, vol. 48, n 3 .

[33] Reynolds G. et C. Walters (2008), "The Use of Customer Surveys for Market Definition and the Competitive Assessment of Horizontal Mergers", Journal of Competition Law and Economics, vol. 4, n ${ }^{\circ} 3$, p. 411-431.

[34] Shapiro C. (1996), "Mergers with Differentiated Products", Antitrust, vol. $10, \mathrm{n}^{\mathrm{o}} 2$, p. $23-30$.

[35] Simpson J.D. et C.T. Taylor (2008), "Do Gasoline Mergers Affect Consumer Prices? The Marathon Ashland Petroleum and Ultramar Diamond Shamrock Transaction", Journal of Law and Economics, vol. 51, n 1, p. 135-152.

[36] Singh N. et X. Vives (1984), "Price and Quantity Competition in a Differentiated Duopoly", RAND Journal of Economics, vol. 15, n 4, p. 546-554.

[37] Taylor C.T. et D.S. Hosken (2007), "The Economic Effects of the Marathon - Ashland Joint Venture : The Importance of Industry Supply Shocks and Vertical Market Structure", Journal of Industrial Economics, vol. 55, $\mathrm{n}^{\circ} 3$, p. $419-451$.

[38] Tenn S. (2008), "The Price Effects of Hospital Mergers : A Case Study of the Sutter-Summit Transaction", Document de travail, $\mathrm{n}^{\circ}$ 293, FTC, Bureau of Economics.

[39] Tschantz S., P. Crooke et L.M. Froeb (2000), "Mergers in Sealed versus Oral Auctions", International Journal of the Economics of Business, vol. 7, $\mathrm{n}^{\circ} 2$, p. 201-212.

[40] Tucker D.S., S.L. Reiter et K.L. Yingling (2007), "The Customer is Sometimes Right : The Role of Costumer Views in Merger Investigations", Journal of Competition Law and Economics, vol. 3, no 4, p. 551-607.

[41] Waehrer K. et M.K. Perry (2003), "The Effects of Mergers in Open-Auction Markets", RAND Journal of Economics, vol. 34, n 2, p. 287-304.

[42] Werden G.J. et L.M. Froeb (1994), "The Effects of Mergers in Differentiated Products Industries : Logit Demand and Merger Policy", Journal of Law, Economics, and Organization, vol. 10, n ${ }^{\circ}$ 2, p. 407-437.

[43] Werden G.J. et L.M. Froeb (2008), "Unilateral Competitive Effects of Horizontal Mergers" in Handbook of Antitrust Economics. P. Buccirossi éd. Cambridge, MA. : MIT Press, p. 43-104.

[44] Whinston M.D. (2006), Lectures on Antitrust Economics, Cambridge, MA : MIT Press. 